\title{
Low-lying excitations in superconducting bilayer systems
}

\author{
Marius Grigorescu \\ Department of Physics and Astronomy \\ University of Western Ontario \\ London, Ontario, Canada N6A 3K7
}

\begin{abstract}
:
The ground and first excited state of two superconducting layers in interaction are studied considering two different coupling terms, one represented by the standard Josephson interaction, and one new, which is a superexchange pairing force between bilayer pairs. It is shown that a moderate-to strong Josephson interaction produces a low-lying collective state, pictured as an out-of-phase oscillation of the BCS gauge angles of the two layers. This antisymmetric angular oscillation might explain the $41 \mathrm{meV}$ resonance observed in the neutron scattering experiments. The bilayer pairs are formed by electrons from different layers with an antiparallel orientation of the spins, being related to the antiferromagnetic arrangement. The pair operators within the layers together with the bilayer pairs generate by commutation an so(5) algebra. It is shown that the transition between the superconducting and antiferromagnetic phases can be explained assuming the dependence on concentration of the bilayer pairing strength, with maximum at half-filling.
\end{abstract}

PACS numbers: 71.10.Li, 74.50.+r, 74.72.-h, 03.65.Fd 


\section{Introduction}

Since the discovery of the high- $\mathrm{T}_{c}$ superconductivity [1] the remarkable properties of the cuprate compounds have been a subject of thorough experimental and theoretical investigation. Although a generally accepted theory is not yet established, the extensive knowledges accumulated during the years indicate that there are few key elements closely related to the mechanism of the high- $\mathrm{T}_{c}$ superconductivity $2 \pi$.

A common element in all cuprate compounds is the layered structure, consisting of $\mathrm{CuO}$ planes separated by block layers [3]. There is also a single parameter, the occupation fraction, whose variation may switch the state between superconducting (SC) and antiferromagnetic (AF). For example, when the planar $\mathrm{Cu}$ atoms of $\mathrm{La}_{2} \mathrm{CuO}_{4}$ or $\mathrm{YBa}_{2} \mathrm{Cu}_{3} \mathrm{O}_{6}$ are divalent each site contains one particle (half-filling), and the compounds are AF insulators. However, if an increasing number of $\mathrm{Cu}$ atoms become trivalent, (hole doping), the AF order is destroyed, and after a transitory spin-glass state the compounds becomes metallic, or below $\mathrm{T}_{c}$, superconducting 4 .

Although the pair binding energy is supposed to come mainly from the interactions within the planes, the interlayer coupling is particularly important. Thus, the properties of the high- $\mathrm{T}_{c}$ compounds become more isotropic in the SC state [2], and the measurements indicate that the current normal to the planes is of Josephson type [5]. In the insulating phase, the AF ordering is not restricted to the nearest neighbors within the planes. It appears also between the adjacent layers [6, 7], and is due to a weak AF interlayer exchange interaction [8, 9].

An interlayer coupling of Josephson type can amplify the pairing correlation within the $\mathrm{CuO}$ planes, explaining the high $\mathrm{T}_{c}$-values and the condensation energy 110, 11]. With this coupling the layered cuprates resemble a stack of Josephson junctions [12], each of them being represented by two adjacent layers (bilayer) coupled by the Josephson interaction.

The low-lying spectrum of the bilayer system is of particular interest, and when the layers become superfluid one can expect collective excitations related to the phase of the BCS (Bardeen-Cooper-Schreiffer) order parameter [13, 11]. As it was shown for the first time in [14], a time-dependent meanfield treatment (TDMF) of the Josephson coupling in a two-component SC system indicates the occurrence of a low-lying resonance. This is antisymmetric between the two components, and can be pictured as an oscillation of the relative phase angle between the two BCS condensates. However, the TDMF approximation is quasi-classical, and therefore in this approach purely quantum effects as tunneling and quantum coherence oscillations are usually suppressed 15.

The relevance of the TDMF phase angle resonance for the low-lying spectrum of a SC bilayer system will be studied in Sect. I. Each component of the bilayer will be described by a reduced BCS interaction term, and spectrum is 
calculated numerically as a function of the strength of the Josephson coupling and the occupation fraction. It will be shown that for moderate-to-strong coupling the phase angle resonance gives a very good description of the first excited state. This resonance is antisymmetric between the planes, appears only in the superconducting state, is coherent between the $\mathrm{CuO}$ planes and of nonmagnetic origin. By these properties it can provide an explanation for the $41 \mathrm{meV}$ resonance observed in the neutron scattering experiments on $\mathrm{YBa}_{2} \mathrm{Cu}_{3} \mathrm{O}_{7}$ [16, 17].

If the two layers can exchange particles, then a pair of electrons localized in one plane may be changed into a pair of electrons from different planes. Such "bilayer pairs" appear also when the Josephson coupling term between the two layers is derived from a general two-body pairing interaction. Because these pairs contain electrons from different planes with an antiparallel arrangement of the spins, they can be naturally related to the AF interlayer ordering. Following the example presented in 14, the extension of the $\mathrm{su}(2) \oplus \mathrm{su}(2)$ algebra of the BCS pairing operators within the planes by the bilayer pair operators leads to the so(5) algebra, and it will be presented in Sect. III.

A model of the high- $\mathrm{T}_{c}$ superconductors where so(5) is generated by the three operators of the total spin, the particle number operator, and six pair operators which generate "rotations" between the total SC order parameter and the Néel vector was formulated in [18, 19]. The AF state at half-filling appears as a solid formed by Cooper pairs, and AF-SC transition corresponds to the melting of this solid under the influence of a fictious "B-field", represented by the chemical potential [18]. However, in this approach the separation of the system in the superconducting components associated to the $\mathrm{CuO}$ planes is not explicit, and it is difficult to establish the connection between the model parameters and the strength of the interlayer Josephson coupling. Therefore, the $41 \mathrm{meV}$ excitation observed in $\mathrm{YBa}_{2} \mathrm{Cu}_{3} \mathrm{O}_{7}$ appears as a Goldstone mode associated to the breaking of the $\mathrm{SO}(5)$ symmetry rather than to the "restoring force" determined by the Josephson interaction, and its generators have both $\mathrm{SC}$ and $\mathrm{AF}$ components [20].

The pairing interaction between the bilayer pairs considered here resemble the superexchange interlayer interaction [2], and is complementary to the Josephson coupling. The properties of the low-lying spectrum of the bilayer system will be studied as a function of the strength of this interaction and the occupation fraction in Sect. IV. It will be shown that by contrast to the Josephson coupling, the new interaction may suppress the superconductivity of the planes, leading to an AF arrangement. A summary of this study and the conclusions are presented in Sect. V. 


\section{Low-lying excitations induced by the Josephson coupling}

The model Hamiltonian considered here is supposed to describe the electrons of two close $\mathrm{CuO}$ layers (bilayer system), denoted $A$ and $B$. Assuming that these layers are related by a translation along the vector $Q$, between their lattice sites there is a one-to-one correspondence expressed by $b=a+Q$. The electrons of each $\mathrm{CuO}$ plane are supposed to interact strongly by pairing forces, and within a restricted BCS approximation, the Hamiltonian has the form

$$
H_{0 L}=\epsilon N_{L}-G_{L} P_{L}^{\dagger} P_{L}, L=A, B .
$$

Here

$$
N_{A}=\sum_{a}\left(c_{a \uparrow}^{\dagger} c_{a \uparrow}+c_{a \downarrow}^{\dagger} c_{a \downarrow}\right), N_{B}=\sum_{a}\left(c_{a+Q \uparrow}^{\dagger} c_{a+Q \uparrow}+c_{a+Q \downarrow}^{\dagger} c_{a+Q \downarrow}\right),
$$

are the particle number operators,

$$
P_{A}^{\dagger}=\sum_{a, a^{\prime}} \Phi\left(a-a^{\prime}\right) c_{a \uparrow}^{\dagger} c_{a^{\prime} \downarrow}^{\dagger}, \quad P_{B}^{\dagger}=\sum_{a, a^{\prime}} \Phi\left(a-a^{\prime}\right) c_{a+Q \uparrow}^{\dagger} c_{a^{\prime}+Q \downarrow}^{\dagger},
$$

create electron pairs with the wave function $\Phi\left(a-a^{\prime}\right)=\Phi\left(a^{\prime}-a\right)$, and $c_{a m}^{\dagger}$ $\left(c_{a m}\right)$ are the creation (annihilation) operators for an electron at the site $a$ $(a+Q)$ of the lattice $A(B)$ with the Z-component of the spin up $(m=\uparrow)$ or down $(m=\downarrow)$. The layers are coupled by a Josephson interaction term [10]

$$
H_{J}=-G_{J}\left(P_{A}^{\dagger} P_{B}+P_{B}^{\dagger} P_{A}\right),
$$

such that the total Hamiltonian has the form

$$
H=\epsilon_{A} N_{A}+\epsilon_{B} N_{B}-G_{A} P_{A}^{\dagger} P_{A}-G_{B} P_{B}^{\dagger} P_{B}-G_{J}\left(P_{A}^{\dagger} P_{B}+P_{B}^{\dagger} P_{A}\right) .
$$

The model parameters are the single-particle energies $\epsilon_{A}, \epsilon_{B}$, the strengths $G_{A}, G_{B}, G_{J}$ of the pairing forces and the number of the lattice sites.

The commutation relations between the pair creation operators and their Hermitian conjugates are determined by the orbital wave function $\Phi\left(a-a^{\prime}\right)$. For a $d_{x^{2}-y^{2}}$-pair field, this function can be expressed as [4]

$$
\Phi\left(a-a^{\prime}\right)=\frac{1}{2}\left(\delta_{a-a^{\prime}, x}+\delta_{a-a^{\prime},-x}-\delta_{a-a^{\prime}, y}-\delta_{a-a^{\prime},-y}\right)
$$

where $x, y$ are vectors along the $\mathrm{x}$ and $\mathrm{y}$ axes of the plane lattice relating the nearest neighbors. The commutator $\left[P_{A}^{\dagger}, P_{A}\right]$ contains the sum

$$
\sum_{a_{1}} \Phi\left(a-a_{1}\right) \Phi\left(a_{1}-a^{\prime}\right)=\delta_{a, a^{\prime}}+\frac{1}{4}\left(\delta_{a-a^{\prime}, 2 x}+\delta_{a-a^{\prime},-2 x}+\delta_{a-a^{\prime}, 2 y}\right.
$$




$$
\left.+\delta_{a-a^{\prime},-2 y}-2 \delta_{a-a^{\prime}, x+y}-2 \delta_{a-a^{\prime},-x-y}-2 \delta_{a-a^{\prime}, x-y}-2 \delta_{a-a^{\prime},-x+y}\right) .
$$

Here only the first term of the right-hand side, which is diagonal, relates nearest neighbors. Assuming that the contribution due to the other terms can be neglected, and that the number of lattice sites $\Omega$ is the same for $A$ and $B$, then $\left[P_{L}^{\dagger}, P_{L}\right]=N_{L}-\Omega, L=A, B$. Defining $P_{0 L}=\left(N_{L}-\Omega\right) / 2$, then $\left[P_{0 L}, P_{L}^{\dagger}\right]=P_{L}^{\dagger}$, and each set of three operators $\left(P_{L}^{\dagger}, P_{0 L}, P_{L}\right), L=A, B$, generates an $\mathrm{su}(2)$ algebra.

If $G_{J}=0$ the ground state $\mid g\left(N_{p}\right)>$ of $H$ is non-degenerate when the total number of pairs $N_{p}$ is even, and double degenerate when it is odd. Thus, $\mid g(2 k)>$ can be factorized in a product of two $k$-pair states, one for each layer, as $|g(2 k)>=| A(k)>\mid B(k)>$, where

$$
\left|L(k)>=\sqrt{\frac{k ! \Omega !}{(\Omega-k) !}}\left(P_{L}^{\dagger}\right)^{k}\right| 0>\quad, \quad L=A, B
$$

and $\mid 0>$ is the particle vacuum. When $N_{p}=2 k+1$ the ground-state doublet states are $\left|g_{a}(2 k+1)\right\rangle=\mid A(k+1) B(k)>$ and $\mid g_{b}(2 k+1)>=$ $\mid A(k) B(k+1)>$. The term $H_{J}$ rise the degeneracy between these states, leading to the entanglement of the two planes. When $G_{J}$ is small, the ground and first excited state of the coupled system are

$$
\mid g_{w}(2 k+1)>=\frac{1}{\sqrt{2}}(|A(k+1) B(k)>+| A(k) B(k+1)>),
$$

and

$$
\mid 1_{w}>=\frac{1}{\sqrt{2}}(|A(k+1) B(k)>-| A(k) B(k+1)>) .
$$

The difference between their energies $E_{x}=E_{1}-E_{0}$ is given in this case by the tunnel splitting $E_{t}=2|<A(k+1) B(k)| H_{J}|A(k) B(k+1)>|=$ $2 G_{J} \Omega^{2}(n+\Omega / 2)(1-n+\Omega / 2)$, where $n=N_{p} / 2 \Omega$ denotes the occupation fraction.

This result was compared with the excitation energy of the first level calculated numerically for a bilayer system with $\Omega=12, \epsilon_{A}=\epsilon_{B}=0$ and $G_{A}=G_{B}=1$, as a function of $n$ and $G_{J}$. The first excited state is always antisymmetric between the planes, and when $G_{J}$ is smaller than $\sim 0.05, E_{x}$ shows a strong odd-even effect at the variation of $N_{p}$. This behavior is illustrated for $G_{J}=0.005$ in Fig. 1 (a) (dots joined by dashed lines). When $G_{J}>0.1$, this strong odd-even effect disappears, as it is shown in Fig. 1 (c) for $G_{J}=0.2$. The comparison with $E_{t}$ (Fig. 1 (a,c), solid line) shows a good agreement when $N_{p}$ is odd and $G_{J}$ is small, but for $N_{p}$ even or when $G_{J}$ increase the differences become large, especially at half-filling. This result 
indicates that at moderate-to-strong coupling $\left(G_{J}>0.1\right)$ the tunneling approach to the Josephson interaction is not realistic. It is interesting to note that the range of the $G_{J}$ values when the coupling is "weak" or "strong" depends of $\Omega$. For instance, decreasing $\Omega$ from 12 to 6 , the interval separating the weak and strong coupling regimes changes from $\sim(0.01,0.1)$ to $\sim(0.05,0.2)$.

The pair transfer matrix element between the ground states, $\Delta=G<$ $g\left(N_{p}\right)\left|P_{L}^{\dagger}\right| g\left(N_{p}-1\right)>$ is a measure of the "softness" of the system with respect to the variation of the number of pairs. A high value of $\Delta$ indicates the increase of the two-particle correlations in the ground state, and may signal the transition to a superconducting state. This parameter was calculated for $G_{J}=0.005$ and 0.2 , and the results are represented in Fig. 1 (b) and (d), respectively. Their comparison confirms the increase of the pairing correlations by the interlayer Josephson interaction, and shows that this effect is stronger at half-filling.

When a layer becomes superconducting, its ground state breaks the U(1) symmetry of the Hamiltonian generated by the particle-number (charge) operator, and is well approximated by the BCS function

$$
\left|B C S(\phi, \lambda)>=e^{\left(2 z P_{L}^{\dagger}-2 z^{*} P_{L}\right)}\right| 0>\quad, L=A, B .
$$

Here $\lambda$ is the Fermi energy, $\phi$ is the BCS "gauge angle", $z=\rho e^{-i \phi}$, and $\rho$ is a variable related to the BCS gap $\Delta_{0}=G<B C S(0, \lambda)\left|P_{L}^{\dagger}\right| B C S(0, \lambda)>$ by $\Delta_{0}=(G \Omega / 2) \sin 4 \rho$, and to the occupation fraction $n=<B C S(0, \lambda) \mid$ $N_{L} \mid B C S(0, \lambda)>/ 2 \Omega$ by $n=\sin ^{2} 2 \rho$. The spontaneous breaking of the $\mathrm{U}(1)$ symmetry generates a specific collective phase-space 21] parameterized by the BCS "gauge" angle $\phi$ and the canonically conjugate momentum $p=$ $\Omega n=\Omega / 2-(\epsilon-\lambda) / G$ |14. In the absence of the interlayer coupling, $\phi$ has a linear dependence on time, $\phi(t)=2 \lambda t$, such that the system performs a free rotation in the BCS gauge-space, with the angular velocity $2 \lambda$. This rotation can be interpreted as the Goldstone mode related to the $U(1)$ symmetry breaking, and the classical Hamilton function associated to this evolution is defined by the expectation value of $H_{0 L}$, 14

$$
\mathcal{H}_{0}(\phi, p)=<B C S\left|H_{0 L}\right| B C S>=2\left(\epsilon-G \frac{\Omega}{2}\right) p+G p^{2} .
$$

This expression indicates that the inertial parameter corresponding to the free gauge rotation is $I_{0}=2 / G$.

When the two superfluid layers are coupled by the Josephson interaction term $H_{J}$, the Hamilton function becomes

$$
\mathcal{H}\left(\phi_{A}, p_{A}, \phi_{B}, p_{B}\right)=<B C S_{A} B C S_{B}\left|H_{0 A}+H_{0 B}+H_{J}\right| B C S_{A} B C S_{B}>=
$$


$2\left(\epsilon-G \frac{\Omega}{2}\right)\left(p_{A}+p_{B}\right)+G\left(p_{A}^{2}+p_{B}^{2}\right)-2 G_{J} \sqrt{p_{A} p_{B}\left(\Omega-p_{A}\right)\left(\Omega-p_{B}\right)} \cos \left(\phi_{A}-\phi_{B}\right)$.

In this case, the dynamics of the system consists of a superposition between a uniform rotation of the "center of mass" angle $\left(\phi_{A}+\phi_{B}\right) / 2$, symmetric between the planes, and an oscillation in the relative angle $\left(\phi_{A}-\phi_{B}\right) / 2$, which is antisymmetric. Assuming the same occupation fraction $n$ for both planes, in the harmonic approximation the excitation energy of this mode is [14]

$$
E_{r}=\sqrt{2 k C}
$$

Here $k=2\left(G-G_{J}\right)+G_{J} / n(1-n)$ is the related inertial parameter, and $C=2 G_{J}\left(\Delta_{0} / G\right)^{2}=2 G_{J} \Omega^{2} n(1-n) \approx E_{t}$, is the strength of the restoring force determined by the Josephson coupling. The same excitation energy is obtained when $H_{J}$ is treated within the random-phase approximation (RPA) [22, 23].

The energy $E_{r}$ of the antisymmetric resonance oscillation and the BCS gap $\Delta_{0}$ obtained with the same parameters as used in the numerical calculations are represented by solid lines in Fig. $1(\mathrm{a}, \mathrm{c})$ and $(\mathrm{b}, \mathrm{d})$, respectively. The agreement with the numerical values is very good at moderate to-strong coupling, when $E_{x} \approx E_{r}$ and $\Delta \approx \Delta_{0}$. At weak coupling $E_{r}$ still reproduces the average trend of $E_{x}$, but $\Delta$ is systematically smaller than $\Delta_{0}$ by a factor $\sim 0.83$. Both $\Delta$ and $\Delta_{0}$ measure the pair correlation functions within a single plane. If each plane develops its superconductivity independently, then in the limit $G_{J} \rightarrow 0$ one would expect that $\Delta \rightarrow \Delta_{0}$. However, this is not observed because when $G_{J}$ is very small, at $N_{p}$ odd the coupling between the two planes leads invariably to an entangled ground state of the form $\mid g_{w}>$. Therefore, the one-pair transfer matrix element $\Delta$ is reduced to $1 / \sqrt{2}$ of the value corresponding to an isolated plane. By contrast, at moderate-to-strong coupling the ground state is spread over many configurations, and BCS approximation becomes realistic. This shows that the interlayer Josephson coupling has an important contribution to the occurrence of the superconductivity within the planes.

Beside the "kinetic" term $G\left(p_{A}^{2}+p_{B}^{2}\right)$ determined by the pairing forces, an additional contribution to $\mathcal{H}$ appears from the electrostatic energy between the planes, $E_{e s}=e^{2}\left(p_{A}-p_{B}\right)^{2} d /\left(2 \epsilon_{0} S\right)$ ( [2], p. 124), where $d$ is the interlayer spacing and $S$ the layer surface. With this term $E_{r}$ becomes $\sqrt{2\left(k+k_{e}\right) C}$, where $k_{e}=2 e^{2} d /\left(\epsilon_{0} S\right)$. However, by contrast to $k$, which is size-independent, $k_{e}$ vanishes at the macroscopic limit $S \rightarrow \infty$, and it will be neglected.

The condensation energy and the superconducting energy gap are known from the measurements of the electronic specific heat [24, 25]. For $\mathrm{YBa}_{2} \mathrm{Cu}_{3} \mathrm{O}_{7}$ the zero temperature superconducting gap has the maximum $\Delta_{0}=20 \mathrm{meV}$, and the condensation energy is $U_{c}=0.5 \mathrm{meV} /$ f.u. cell. Assuming that $\Delta_{0}=G \Omega \sqrt{n(1-n)}$, with $n \sim 1 / 2$, and $G_{J} / G \approx 0.5$, one obtains $E_{r} \approx 50$ 
meV. This low value suggests that the antisymmetric angular oscillations in the BCS gauge space presented here may provide an explanation for the 41 meV resonance observed in the neutron scattering experiments [16, 17].

The general form of the operator creating a pair of electrons with a threedimensional orbital wave function $\Psi\left(l-l^{\prime}\right)$ in the bilayer system is

$$
P^{\dagger}=\sum_{l, l^{\prime}} \Psi\left(l-l^{\prime}\right) c_{l \uparrow}^{\dagger} c_{l^{\prime} \downarrow}^{\dagger}
$$

where $l$ runs over the lattice sites of both planes, $A$ and $B$. Assuming that the in-plane section $\Psi\left(a-a^{\prime}\right)$ is the same as $\Phi\left(a-a^{\prime}\right)$, and $\Psi(l)$ is periodic in the $Q$-direction, $\Psi(l)=\Psi(l+Q)$, this general pair creation operator can be decomposed as

$$
P^{\dagger}=P_{A}^{\dagger}+P_{B}^{\dagger}+R^{\dagger}
$$

where $P_{A}^{\dagger}$ and $P_{B}^{\dagger}$ are the pair creation operators within the planes of Eq. (3), and

$$
R^{\dagger}=\sum_{a, a^{\prime}} \Phi\left(a^{\prime}-a\right)\left(c_{a+Q \uparrow}^{\dagger} c_{a^{\prime} \downarrow}^{\dagger}+c_{a+Q \downarrow}^{\dagger} c_{a^{\prime} \uparrow}^{\dagger}\right)
$$

creates a pair of electrons localized in different planes, with an antiparallel spin orientation.

The antiparallel orientation of the nearest spins from different planes is a characteristic of the interlayer antiferromagnetic ordering. In fact, the AF ordering of the high- $\mathrm{T}_{c}$ materials was described within the resonance valence bond (RVB) theory as a condensate of nearest neighbors singlet pairs [26]. A general pairing interaction of the form $-G P^{\dagger} P$ leads naturally the terms of the Hamiltonian $H$, but contains also other terms dues to the "bilayer pairing" operators $R$. In particular, a pairing interaction $-R^{\dagger} R$ between the bilayer pairs can represent a convenient approximation for the superexchange interlayer coupling presented in [2], p. 395. This term can produce the AF condensation, and it will be studied by extending to so(5) the $\mathrm{su}(2) \oplus \operatorname{su}(2)$ algebra used to describe the effect of the interlayer Josephson coupling.

\section{The so(5) algebra of the pair operators in the bilayer system}

The shift along the chain

$$
P_{A}^{\dagger} \rightarrow R^{\dagger} \rightarrow P_{B}^{\dagger}
$$

of the three pair creation operators defined above is generated by commutation with the interlayer "hopping" operator

$$
T_{+}=\sum_{a}\left(c_{a+Q \downarrow}^{\dagger} c_{a \downarrow}-c_{a+Q \uparrow}^{\dagger} c_{a \uparrow}\right),
$$


such that

$$
\left[T_{+}, P_{A}^{\dagger}\right]=-R^{\dagger} \quad, \quad\left[T_{+}, R^{\dagger}\right]=2 P_{B}^{\dagger} \quad, \quad\left[T_{+}, P_{B}^{\dagger}\right]=0
$$

Similarly, the down shift

$$
P_{B}^{\dagger} \rightarrow R^{\dagger} \rightarrow P_{A}^{\dagger}
$$

is generated by commutation with $T_{-} \equiv\left(T_{+}\right)^{\dagger}$. Each set of three operators, $\left(R^{\dagger}, R_{0}, R\right)$ and $\left(T_{+}, T_{0}, T_{-}\right)$, with $R_{0}=P_{0 A}+P_{0 B}$ and $T_{0}=\left(N_{B}-N_{A}\right) / 2$ generate a new su(2) algebra, while the set of the 10 operators $T_{+}, T_{-}, P_{A}^{\dagger}, P_{A}$, $R^{\dagger}, R, P_{B}^{\dagger}, P_{B}, P_{0 A}, P_{0 B}$ generate the so(5) algebra.

It is important to note that by contrast to $P_{A}^{\dagger}$ and $P_{B}^{\dagger}$ which create spin singlet pairs, the pair created by $R^{\dagger}$ is the $m=0$ component of a spin triplet. A spin singlet pair of electrons from different planes is generated by

$$
S^{\dagger}=\sum_{a, a^{\prime}} \Phi\left(a-a^{\prime}\right)\left(c_{a+Q \uparrow}^{\dagger} c_{a^{\prime} \downarrow}^{\dagger}-c_{a+Q \downarrow}^{\dagger} c_{a^{\prime} \uparrow}^{\dagger}\right)
$$

Pairs of this type have been used to construct the RVB vacuum in [27. The shift along the chain

$$
P_{A}^{\dagger} \rightarrow S^{\dagger} \rightarrow P_{B}^{\dagger}
$$

is generated by the commutation with the hopping operator

$$
\tau_{+}=\sum_{a}\left(c_{a+Q \downarrow}^{\dagger} c_{a \downarrow}+c_{a+Q \uparrow}^{\dagger} c_{a \uparrow}\right)
$$

and $\tau_{+}, \tau_{-}, P_{A}^{\dagger}, P_{A}, S^{\dagger}, S, P_{B}^{\dagger}, P_{B}, P_{0 A}, P_{0 B}$ generate also an so(5) algebra.

In the defining representation, the so(5) generators are $5 \times 5$ matrices $f_{p q}=e_{p q}-e_{-q-p}$, where the indices $p$ and $q$ take the values $-2,-1,0,1,2$, and $e_{p q}$ is the $5 \times 5$ matrix with the element on the row $p$ and the column $q$ equal to 1 , and all the rest 0 . Thus $\left[e_{p q}, e_{k l}\right]=\delta_{q k} e_{p l}-\delta_{l p} e_{k q}$, and the commutation relations of the so(5) generators are

$$
\left[f_{p q}, f_{k l}\right]=\delta_{q k} f_{p l}-\delta_{p l} f_{k q}+\delta_{p-k} f_{-l q}-\delta_{-q l} f_{p-k}
$$

The correspondence between the generators with negative roots of the defining representation of so(5) and the two different realizations provided by the pairing operators, denoted $\mathrm{so}(5)_{r}$ and $\mathrm{so}(5)_{s}$, is summarized in the Table 1. One should note that these two realizations are related simply by a change of sign for all the single-particle states with spin up in the layer $A$. This transformation can be written as $c_{a \uparrow}^{\dagger} \rightarrow-c_{a \uparrow}^{\dagger}$, and as long as it leaves the Hamiltonian invariant, the results are independent on the choice of $\mathrm{so}(5)_{r}$ or 
$\mathrm{so}(5)_{s}$ for the explicit realization.

The Cartan subalgebra of so(5) is generated by two elements, $f_{22}, f_{11}$, and in both realizations

$$
f_{22}=\frac{1}{2}\left(P_{0 B}+P_{0 A}\right) \quad, \quad f_{11}=\frac{1}{2}\left(P_{0 B}-P_{0 A}\right) .
$$

The algebra so(5) is semisimple of rank 2 , and its irreducible representations can be labeled by the numbers $\left[N_{1}, N_{2}\right], N_{1} \geq N_{2} \geq 0$ of the associated Young diagram, or by the eigenvalues of the generators of the Cartan subalgebra for the highest weight state [28]. If this state is chosen to be the particle vacuum, $\mid 0>$, then similarly to the case of $\mathrm{su}(3)$ [29], the states $\mid k l m>$

$$
\left|k l m>=\left(f_{2-1}\right)^{k}\left(f_{12}\right)^{l}\left(f_{02}\right)^{m}\right| 0>,
$$

with $k+l+m \leq 2 \Omega$ span the Hilbert space $\mathcal{H}_{\Omega}$, carrier of the symmetric irreducible representation $[\Omega, 0]$ of so(5).

The labeling of the states of this irreducible representation requires three indices. One of them is provided by the total number of particles $2(k+l+m)$, which takes values between 0 and $4 \Omega$, and another by the eigenvalue $k-l$ of $T_{0}\left(=\tau_{0}\right)$. The choice of the third index is a difficult long-standing problem, because the set of all possible states $\mid k l m>$ with a fixed $N$ and $k-l$ is non-orthogonal and overcomplete [30]. For instance, there is a non-vanishing amplitude $<k k 0 \mid 002 k>=(2 k) ! k ! \Omega ! /(\Omega-k)$ ! for recoupling $2 k$ bilayer pairs in two sets of $k$ one-layer pairs.

The basis of the $[\Omega, 0]$ irrep spaces can be obtained by using vector coherent states methods, by projection from a highest weight state 31. Here the basis will be constructed numerically, restricting the overcomplete set of Eq. (27) by the Gramm-Schmidt procedure. As a test, the dimension of the spaces $\mathcal{H}_{\Omega}$ generated for $2 \leq \Omega \leq 12$ was found to be the same as given by the analytical formula

$$
d(\Omega, 0)=\frac{1}{6}(2 \Omega+3)(\Omega+2)(\Omega+1)
$$

The action of the generators on the (non-orthonormal) states of Eq. (27) is expressed by $f_{2-1}|k l m>=| k+1 l m>, f_{21}|k l m>=| k l+1 m>, f_{20} \mid k l m>=$ $\mid k l m+1>$, and

$$
\begin{aligned}
& f_{-12}|k l m>=-k(k+m-\Omega-1)| k-1 l m>+m(m-1) \mid k l+1 m-2> \\
& f_{12}|k l m>=-l(l+m-\Omega-1)| k l-1 m>+m(m-1) \mid k+1 l m-2> \\
& f_{02}|k l m>=k l| k-1 l-1 m+1>-2 m\left(k+l-\Omega+\frac{m-1}{2}\right) \mid k l m-1>.
\end{aligned}
$$


These formulas are useful to calculate the matrix elements of the general pairing Hamiltonian in the so(5) basis.

\section{SC-AF transition induced by the bilayer pair interaction}

The extended Hamiltonian which includes the interaction between the bilayer pairs has the general form

$$
H_{g}=-G\left(P_{A}^{\dagger} P_{A}+P_{B}^{\dagger} P_{B}\right)-G_{J}\left(P_{A}^{\dagger} P_{B}+P_{B}^{\dagger} P_{A}\right)-g R^{\dagger} R .
$$

This Hamiltonian is expressed only in terms of the so( 5$)_{r}$ generators, and therefore it allows to restrict the Hilbert space to a space $\mathcal{H}_{\Omega}$ of irreducible representation.

The low-lying spectrum of $H_{g}$ has been calculated for $G=1, G_{J}=0.5$ and $\Omega=6$, as a function of the strength $g$ of the bilayer pairing interaction and the occupation fraction $n$. The ground state energy $E_{0}$ and the excitation energy of the first level $E_{x}=E_{1}-E_{0}$ are represented as a function of $n$ and $g$ in Fig. 2(a) and 2(b). The pair transfer matrix element $\Delta$ is represented in Fig. 3(a).

For the study of the AF correlations each lattice $L=A, B$ is separated in two sublattices, denoted by $(L, 1)$ and $(L, 2)$, such that the nearest neighbors of the spins from $(L, 1)$ within the plane $L$ are in $(L, 2)$. If the nearest neighbors of $(A, 1),(A, 2)$ outside the $A$-plane are in $(B, 1),(B, 2)$, then in a classical antiferromagnetic configuration the spins from $(A, 1)$ and $(B, 2)$ have all the same orientation, opposite to that of the spins from $(A, 2)$ and $(B, 1)$.

In a quantum system the situation is more complex, because the ground state is a symmetric superposition of classical AF states with opposite orientations of the Néel vector [32]. Thus, as a measure of the AF correlations in the quantum bilayer system here will be considered the ground state ex-

pectation value of the operator $\mathcal{C}=\left(S_{z}^{A}(1)-S_{z}^{A}(2)\right)\left(S_{z}^{B}(1)-S_{z}^{B}(2)\right)$, where

$$
S_{z}^{A}(\alpha)=\frac{1}{2} \sum_{a \in(A, \alpha)}\left(c_{a \uparrow}^{\dagger} c_{a \uparrow}-c_{a \downarrow}^{\dagger} c_{a \downarrow}\right),
$$

and

$$
S_{z}^{B}(\alpha)=\frac{1}{2} \sum_{a \in(B, \alpha)}\left(c_{a+Q \uparrow}^{\dagger} c_{a+Q \uparrow}-c_{a+Q \downarrow}^{\dagger} c_{a+Q \downarrow}\right)
$$

for $\alpha=1,2$ are the $\mathrm{z}$ (c-axis)-components of the total spin for each sublattice. If only the terms containing nearest neighbors are retained, then the groundstate expectation value $<g|\mathcal{C}| g>$ can be approximated by $<g \mid S_{z}^{A}(1) S_{z}^{B}(1)+$ $S_{z}^{A}(2) S_{z}^{B}(2) \mid g>$ and also by $<g\left|S_{z}^{A} S_{z}^{B}\right| g>$, where $S_{z}^{L}=S_{z}^{L}(1)+S_{z}^{L}(2)$. This 
approximation is particularly convenient if the Hilbert space is restricted to $\mathcal{H}_{\Omega}$. In this case, the matrix elements of $S_{z}^{A} S_{z}^{B}$ can be calculated analytically, and have the expression

$$
\begin{gathered}
<k^{\prime} l^{\prime} m^{\prime}\left|S_{z}^{A} S_{z}^{B}\right| k l m>=\left[\left(k+\frac{m}{2}\right)\left(l+\frac{m}{2}\right)-k l-m \frac{k+l+m}{2}+\right. \\
\left.\frac{m(m-1)}{2} \frac{\left(\Omega^{2}-1\right)}{\Omega(2 \Omega-1)-1}\right] f+\frac{m(m-1)(\Omega-1)}{\Omega(2 \Omega-1)-1} g,
\end{gathered}
$$

where $f=<k^{\prime} l^{\prime} m^{\prime} \mid k l m>$ and $g=<k^{\prime} l^{\prime} m^{\prime} \mid k+1 l+1 m-2>$.

The expectation value of this operator in the ground state of $H_{g}$ is represented as a function of $n$ and $g$ in Fig. 3(b). The results indicate that when $g$ is small $\Delta>0,<g\left|S_{z}^{A} S_{z}^{B}\right| g>=0$, and the main components of the ground state are the states $\mid k l m>$ with $k+l>m$. However, when $g$ increases above $\sim 0.8$ the structure of the ground state has a sharp transition, and the states $\mid k l m>$ with $m>k+l$ become dominant. During this transition $\Delta$ vanishes (Fig. 3 (a)) and $<g\left|S_{z}^{A} S_{z}^{B}\right| g>$ becomes negative (Fig. 3 (b)), indicating the onset of the AF ground-state correlations. In the transition region the excitation energy $E_{x}$ has maxima when the number of pairs is even, and minima when it is odd (Fig. 2(b)). This behavior is correlated with the change in the structure of the first excited state, whose main components can be obtained by turning symmetrically one bilayer pair of the ground state into a pair within the layers.

\section{Summary and conclusions}

The interlayer coupling in the cuprate compounds has a fundamental role in the physics of the high- $\mathrm{T}_{c}$ superconductivity, and in the recent years an increasing experimental and theoretical effort was devoted for understanding its mechanism [2]. In this work the low-lying properties of two superconducting layers in interaction have been described considering two different coupling terms, one represented by the standard Josephson interaction, and one new, which is a "superexchange" pairing force between bilayer pairs.

The Josephson coupling between two superconductors is usually presented as a perturbation allowing the flow of the Josephson tunneling current [33]. Complementary to this result, a TDMF [14] or RPA [22] calculation indicates that with this interaction the difference between the BCS phase angles of the two superconductors is not a constant, but oscillates in time, producing an excitation which could be observed as an antisymmetric low-lying resonance. These two pictures have been compared (Fig. 1) with the exact results obtained numerically for a restricted BCS Hamiltonian.

It was shown that the tunneling approach describes very well the energy 
of the first excited state when the number of pairs is odd and the strength of the Josephson coupling is small. In this case the bare ground state is double degenerate. The Josephson interaction rises this degeneracy, splitting the doublet into a non-degenerate ground state, symmetric between the two components, and the first excited state, which is antisymmetric. The two components of the bare ground state become non-stationary states, interchanged periodically in time by quantum coherence oscillations. These oscillations can be pictured as a periodic exchange of a pair between the two layers. One should note, however, that at the relatively high temperature of the cuprate superconductors they might be completely suppressed by thermal decoherence 34].

At moderate-to-strong coupling the excitation energy of the first level is very well described as a collective out-of-phase oscillation of the BCS gauge angles of the two layers. By contrast to the phase of a quantum wave function, the BCS angle is a collective variable defined only in the superconducting state, canonically conjugate to the particle number 114, 29. Assuming a ratio $G_{J} / G=0.5$ between the strength of the Josephson coupling $G_{J}$ and the pairing force within the layers $G$, the excitation energy of this resonance in $\mathrm{YBa}_{2} \mathrm{Cu}_{3} \mathrm{O}_{7}$ is $\sim 50 \mathrm{meV}$. This is a promising result, because indicates that by a more accurate estimate of $G_{J} / G$, the antisymmetric angular oscillation in the BCS gauge space might explain the $41 \mathrm{meV}$ resonance observed in the neutron scattering experiments [16, 17].

The effect of the Josephson coupling on the two-particle correlations was studied by calculating the pair transfer matrix element $\Delta$ between ground states. An increase of $\Delta$ indicates that the structure of the ground state is less sensitive to the addition or removal of a pair, and becomes closer to the one of the BCS condensate. The results presented in Fig. 1 (b) and (d) show that the Josephson coupling can induce the configuration mixing required for the occurrence of the superconductivity within the layers. At moderate-tostrong coupling $\Delta$ is very close to the BCS gap (Fig. $1(\mathrm{~d})$ ), indicating that the ground state of the whole system can be well approximated by a product of two independent BCS functions, one for each plane.

The decomposition of the most general pairing interaction in a bilayer system leads naturally to the Josephson coupling term, but also to a pairing interaction between "bilayer pairs", similar to the superexchange force discussed in ref. [2]. These pairs are formed by electrons from different planes with an antiparallel orientation of the spins, and therefore are related to the AF arrangement. The three different pair creation operators, (two for pairs within the layers and one for the bilayer pairs), together with their Hermitian conjugates generate by commutation the so(5) algebra. This result is important, because it shows that for a wide class of pairing Hamiltonians the many-body Hilbert space can be split in a series of finite-dimensional spaces of so(5) irreducible representation. In particular, one can expect to find the ground state within a space $\mathcal{H}_{\Omega}$, carrier of a symmetric irrep of so(5). The 
construction of these spaces is a non-trivial problem in the Lie algebra representation theory, and it was presented in Sect. III.

The low-lying properties of two superconducting layers coupled by Josephson and bilayer pair interactions have been studied as a function of the occupation fraction in Sect. IV. For the explicit realization was used a set of $\mathrm{so}(5)_{r}$ operators, but this choice is irrelevant as long as the Hamiltonian contains only a single type of bilayer pairs $(R$ or $S)$, and is invariant to the $\mathrm{so}(5)_{r} \leftrightarrow \mathrm{so}(5)_{s}$ transformation. The results indicate that when the strength of the bilayer pair interaction $g$ is $\sim 0.8 G$ or greater, there is a strong oddeven effect in the excitation energy of the first state (Fig. 2(b)), and the superconductivity within the planes disappears (Fig. 3 (a)). This transition is accompanied also by the onset of an AF arrangement between the spins of the two layers (Fig. $3(\mathrm{~b})$ ), and is practically independent of the occupation fraction. Therefore, within this model an AF-SC transition at the variation of $n$ can be explained only by assuming that the ratio $g / G$ depends on $n$ and reaches the maximum at half-filling. The increase of the pairing interaction strengths within the planes at half-filling was noticed in ref. [35. The study of the concentration dependence of the interlayer coupling strengths appears therefore as a subject worth of further investigation.

\section{Acknowledgements}

The hospitality of Prof. M. Singh and of the Department of Physics and Astronomy, University of Western Ontario, is gratefully acknowledged. Thanks are due to Prof. D. J. Rowe, University of Toronto, for many stimulating discussions.

\section{References}

[1] J. G. Bednorz and K. A. Müller, Z. Phys. B 64, 189 (1986).

[2] P. W. Anderson, The Theory of Superconductivity in the High-T Cuprates, Princeton, 1997.

[3] M. Imada, A. Fujimori and Y. Tokura, Rev. Mod. Phys. 70, 1039 (1998).

[4] D. J. Scalapino, Phys. Rep. 250, 329 (1995). 
[5] L. M. Fisher, A. V. Kalinov, S. E. Savel'ev, I. F. Voloshin and V. A. Yampol'skii, Physica C 309, 284 (1998).

[6] D. Vaknin, S. K. Sinha, D. E. Moncton, D. C. Johnston, J. M. Newsam, C. R. Safinya and H. E. King, Jr., Phys. Rev. Lett. 58, 2802 (1987).

[7] T. Freltoft, J. E. Fischer, G. Shirane, D. E. Moncton, S. K. Sinha, D. Vaknin, J. P. Remeika, A. S. Cooper and D. Harshman, Phys. Rev. B36, 826 (1987).

[8] M. A. Kastner, R. J. Birgeneau, T. R. Thurston, P. J. Picone, H. P. Jenssen, D. R. Gabbe, M. Sato, K. Fukuda, S. Shamoto, Y. Endoh, K. Yamada and G. Shirane, Phys. Rev. B38, 6636 (1988).

[9] R. P. Singh and M. Singh, Phys. Rev. B46, 14069 (1992).

[10] S. Chakravarty, A. Sudbo, P. W. Anderson and S. Strong, Science 261, 337 (1993).

[11] P. W. Anderson, Science 268, 1154 (1995).

[12] M. Tinkham, Physica C235-240, 3 (1994).

[13] M. I. Salkola and J. R. Schrieffer, Phys. Rev. B 58, R5944 (1998-II).

[14] M. Grigorescu, J. Phys. G 16, 417 (1990).

[15] M. Grigorescu, Rom. Journ. Phys. 42, 509 (1997).

[16] H. F. Fong, B. Keimer, P. W. Anderson, D. Reznik, F. Dogan and I. A. Aksay, Phys. Rev. Lett. 75, 316 (1995).

[17] H. F. Fong, B. Keimer, D. Reznik, D. L. Milius and I. A. Aksay, Phys. Rev. B 54, 6708 (1996).

[18] S. C. Zhang, Science 275, 1089 (1997).

[19] S. Rabello, H. Kohno, E. Demler and S. C. Zhang, Phys. Rev. Lett. 80, 3586 (1998).

[20] C. L. Henley, Phys. Rev. Lett. 80, 3590 (1998).

[21] M. Grigorescu, in Frontier Topics in Nuclear Physics, edited by W. Scheid and A. Sandulescu (Plenum Press, New York, 1994), p. 491.

[22] M. Grigorescu and E. Iancu, Z. Phys. A337, 139 (1990).

[23] M. Grigorescu, Rev. Roum. Phys. 37, 107 (1992). 
[24] J. W. Loram, K. A. Mirza and P. F. Freeman, Physica C171, 243 (1990).

[25] J. W. Loram, K. A. Mirza, J. R. Cooper and J. L. Tallon, Physica C 282, 1405 (1997).

[26] P. W. Anderson, Science 235, 1196 (1987).

[27] R. Eder, A. Dorneich, M. G. Zacher, W. Hanke, S. C. Zhang, Phys. Rev. B59, 561 (1999).

[28] D. J. Rowe, J. Math. Phys. 35, 3163, 3178 (1994).

[29] M. Grigorescu, St. Cerc. Fiz. 36, 3 (1984).

[30] K. T. Hecht, Nucl. Phys. A 102, 11 (1967).

[31] D. J. Rowe and K. T. Hecht, J. Math. Phys. 36, 4711 (1995).

[32] M. Grigorescu and M. R. Singh, Phys. Rev. B 59, 6013 (1999).

[33] C. Kittel, Introduction to Solid State Physics, 5th ed., Wiley, New York, (1976), p. 390.

[34] M. Grigorescu, Physica A 256, 149 (1998).

[35] K. P. Sinha and M. Singh, J. Phys. C 21, L231 (1988), Phys. Stat. Sol. 159, 787 (1990).

Table 1. The so(5) generators with negative roots

\begin{tabular}{|c|c|c|c|c|}
\hline $\mathrm{so}(5)$ & $f_{21}$ & $f_{20}$ & $f_{2-1}$ & $f_{10}$ \\
\hline $\mathrm{so}(5)_{r}$ & $P_{A}^{\dagger}$ & $R^{\dagger} / \sqrt{2}$ & $P_{B}^{\dagger}$ & $T_{+} / \sqrt{2}$ \\
\hline $\mathrm{so}(5)_{s}$ & $-P_{A}^{\dagger}$ & $S^{\dagger} / \sqrt{2}$ & $P_{B}^{\dagger}$ & $\tau_{+} / \sqrt{2}$ \\
\hline
\end{tabular}

\section{Figure Captions}

Fig. 1. The excitation energy $E_{x}=E_{1}-E_{0}(\mathrm{a}, \mathrm{c})$ and the pair transfer matrix element $\Delta=G<g\left(N_{p}\right)\left|P_{A}^{\dagger}\right| g\left(N_{p}-1\right)>(\mathrm{b}, \mathrm{d})$ as a function of the occupation fraction $n=N_{p} / 2 \Omega$ when $\Omega=12, G_{A}=G_{B}=1, g=0$ (dots joined by dashed lines). For comparison, $E_{r}, E_{t}$ and $\Delta_{0}$ are represented by solid lines. Two values of the Josephson coupling strength are considered, 
$G_{J}=0.005(\mathrm{a}),(\mathrm{b})$ and $G_{J}=0.2(\mathrm{c}),(\mathrm{d})$.

Fig. 2. The ground state energy $E_{0}$ (a) and the excitation energy $E_{x}=E_{1}-E_{0}(\mathrm{~b})$ as a function of the occupation fraction $n$ and the strength of the bilayer pairs interaction $g$ when $\Omega=6, G_{A}=G_{B}=1$ and $G_{J}=0.5$.

Fig. 3. The pair transfer matrix element $\Delta$ (a) and the correlation function $<g\left(N_{p}\right)\left|S_{z}^{A} S_{z}^{B}\right| g\left(N_{p}\right)>($ b) as a function of the occupation fraction $n$ and the strength of the bilayer pairs interaction $g$ when $\Omega=6, G_{A}=G_{B}=$ 1 and $G_{J}=0.5$. 

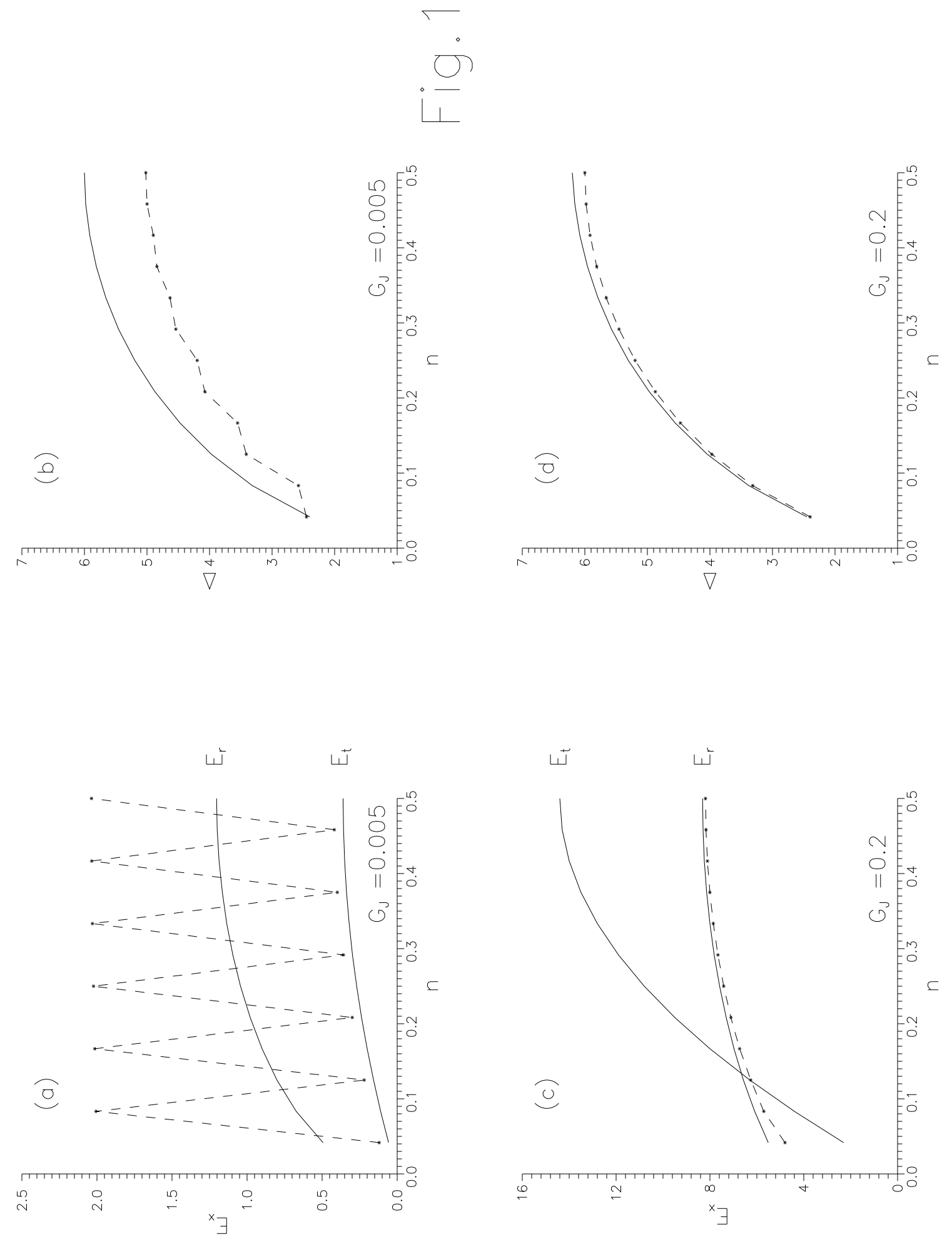

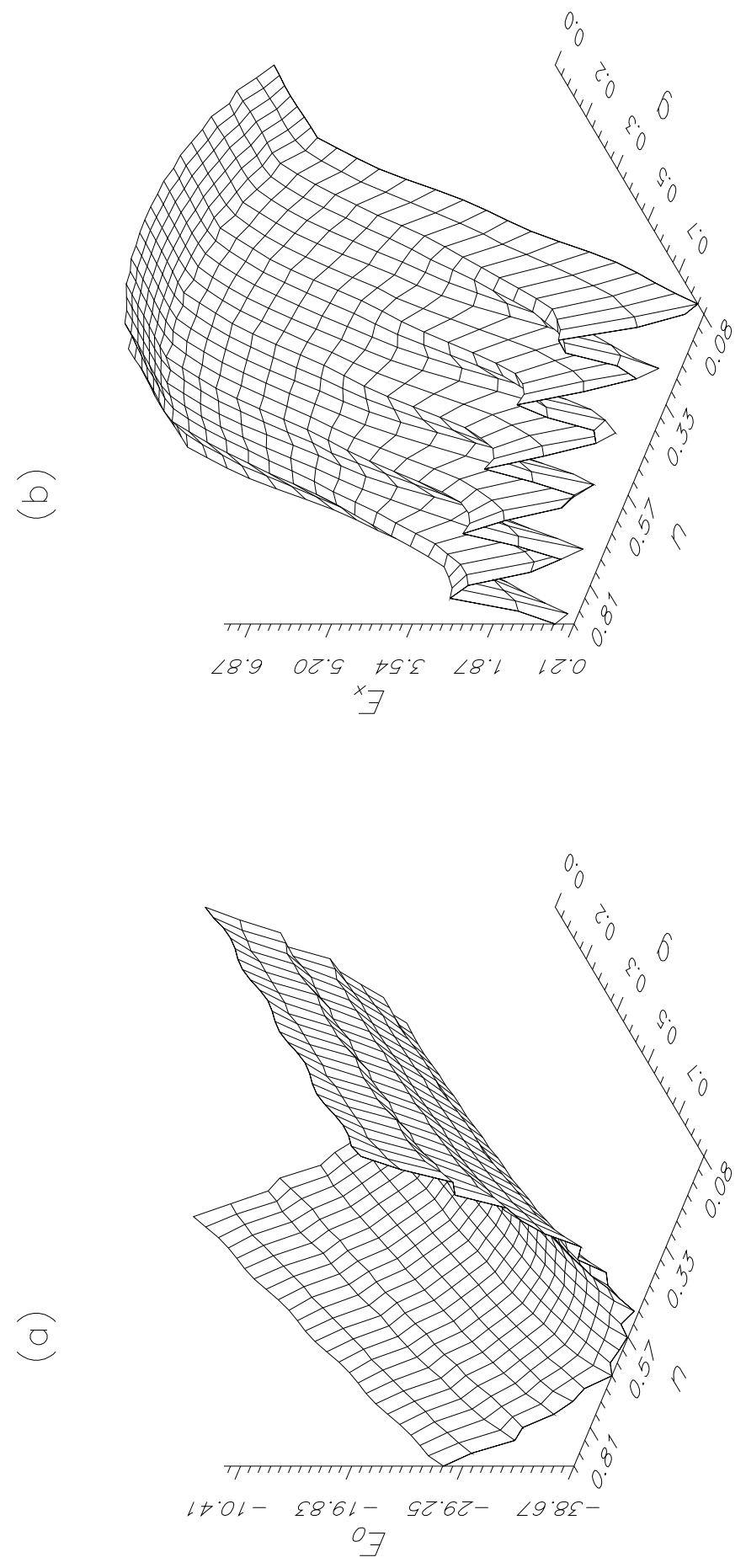

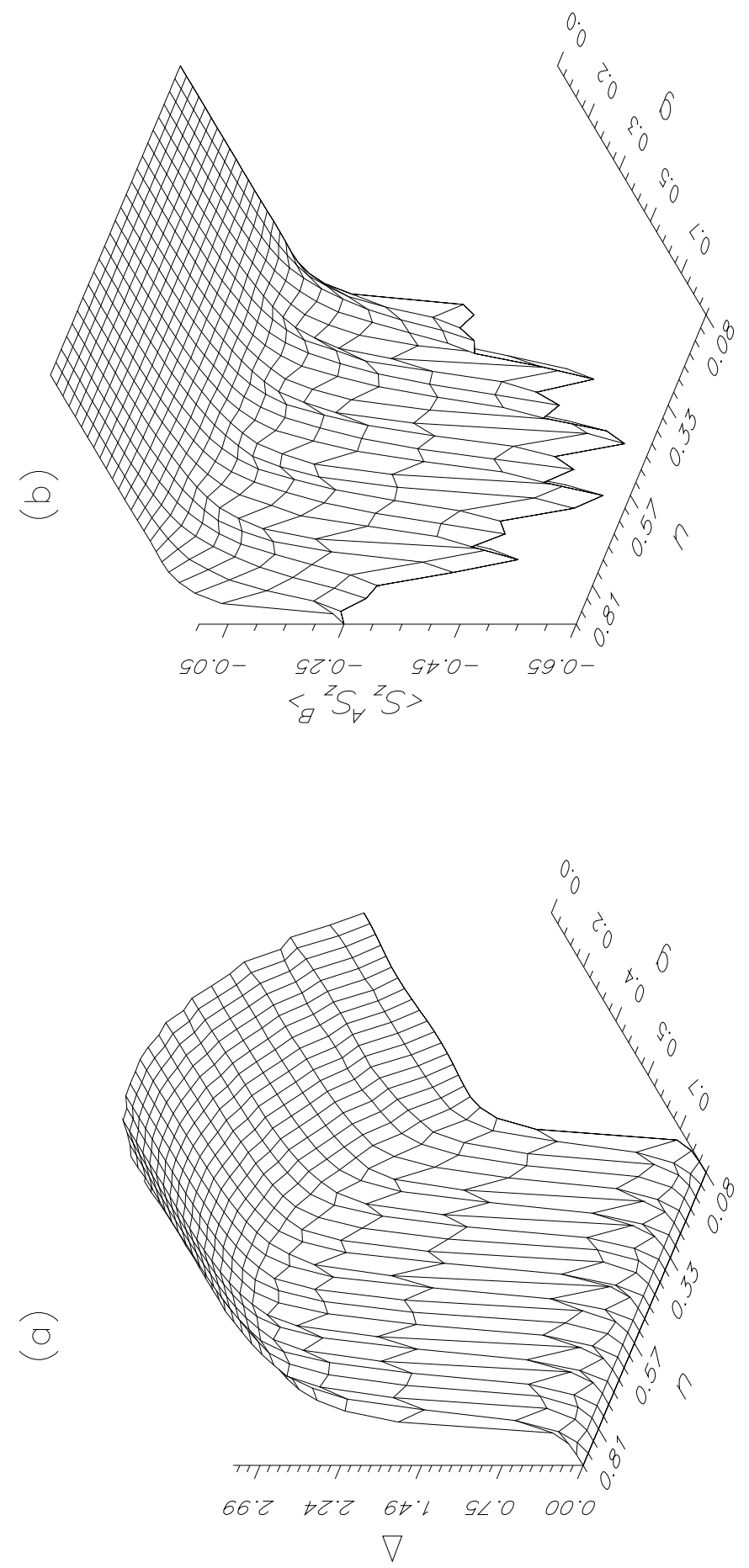